\title{
Benthic Algal Community in Relationship to Perturbation in the Tiko Mangrove Estuary Cameroon
}

\author{
Godlove Ambe Neba*, Neculina Anyinkeng, Coleen Mumbang, Ambo Beatrice Fonge \\ Department Plant Science, Faculty of Science, University of Buea, Buea, Cameroon \\ Email: ${ }^{\star}$ negoda70@yahoo.com
}

How to cite this paper: Ambe Neba, G., Anyinkeng, N., Mumbang, C. and Fonge, A.B. (2021) Benthic Algal Community in Relationship to Perturbation in the Tiko Mangrove Estuary Cameroon. Open Journal of Ecology, 11, 540-564.

https://doi.org/10.4236/oje.2021.117035

Received: June 3, 2021

Accepted: July 23, 2021

Published: July 26, 2021

Copyright (อ 2021 by author(s) and Scientific Research Publishing Inc. This work is licensed under the Creative Commons Attribution International License (CC BY 4.0).

http://creativecommons.org/licenses/by/4.0/

(c) (i) Open Access

\begin{abstract}
Mangrove forests are among the most productive natural ecosystems in the world and offer invaluable ecological functions, yet are seriously threatened. Anthropogenic activities within mangroves can cause alteration of physicochemical parameters of sediments which in turn affect the assemblage of biological communities. This study was designed to assess the impact of anthropogenic disturbances within the Tiko mangroves on the benthic algal community. Perturbations were assessed through questionnaires. Two sets of composite sediment samples were collected for physicochemical and algal analyses from seven sites within the mangroves following standard methods. Seven drivers of change were identified (agriculture, human settlement, logging, fuel wood collection, fishing, fish smoking and petty trading) as the main perturbations of the ecosystem. A total of 141 benthic species were identified at the different sites. The Bacillariophyta were the most abundant with 60 algal species followed by Chlorophyta with 38 algal species. The most dominant species were Chlorobotrys regularis (50) and Microcytis sp (38). Site 3 (settlement site) was the most diversed with 72 species. The mean abundance of benthic algae ranged from $0.59 \pm 0.17$ to $2.08 \pm 0.58$. High concentrations of nutrients and the presence of Bacillariophyta and Microcystis suggest pollution. There is a need to monitor activities carried out around the mangrove ecosystem with respect to quantity and quality of waste discharged into the environment since this is a highly sensitive environment.
\end{abstract}

\section{Keywords}

Benthic Algal Community, Sediments, Perturbation, Tiko Mangrove, Cameroon 


\section{Introduction}

Mangrove forests are composed of unique plant species (halophilic trees and shrubs) that have specific morphological, physiological, and reproductive characteristics which enable them to survive in a critical interface among terrestrial, estuarine, and near-shore marine ecosystems in tropical and subtropical regions around the world [1]. They are among the most productive natural ecosystems in the world as they offer invaluable ecological functions due to their exceptional flora and fauna diversities [2]. Despite these critical functions, globally, mangrove forests are the most threatened tropical ecosystem and are being degraded and depleted at alarming rates [3]. Industrial activity, population growth, human migration to coastal areas, wood and pole extraction, fish smoking, unregulated aqua-culture development and poor management practices stress these systems [4]. Sediment pollution, as a result of human activities, can cause alteration of physicochemical parameters of sediments which in turn affects the assemblage of biological communities residing in them [5]. As a primary source of energy in the aquatic food weds, algae are used as food by many organisms living in the mangrove sediment and support various communities of small benthic animals such as nematode worms, bivalves, copepods, polychaetes, cetacean, mollusk and soldier crab [6]. They represent a wide variety of evolutionary traits, life-forms, and strategies belonging to several classes. Among the different algal groups, diatoms (Bacillariophyta) are the most abundant, contributing $20 \%$ of the global primary productivity. Consequently, they are the most preferred food for water organisms and facilitate biogeochemical processes for different elements particularly for global cycling of silica and carbon [7]. Hendrarto and Nitisupario [6] reported that, in the tropical mangrove forest, benthic algae are one of the important micro-flora living in the sediment. Algae have responded to variations in water chemistry, season, and physiography variables [8]. Studies conducted in tropical and subtropical Africa indicated that the community assemblages of diatoms were impacted by human induced water quality alterations [5] [9] [10] [11] [12]. Being microscopic, they are the first to sense and respond to environmental changes. Algae have been recognized as important indicators of water and sediment pollution [13].

Studies on mangroves in Cameroon have mainly focused on quantifying mangrove logging [2] [14] [15] [16] [17] and the implications of wood exploitation for fish smoking [3] [18]. Reports on anthropogenic activities and their impact on physicochemical properties of mangrove sediments and benthic algal communities in this area are scarce. This study was designed to assess the impact of anthropogenic activities within the Tiko mangroves on their benthic algal community.

\section{Materials and Methods}

\subsection{Description of Study Site and Selection of Sampling Sites}

The study was carried out within the mangroves of Tiko, located between lati- 
tude $4^{\circ} 28^{\prime} 30^{\prime \prime} \mathrm{N}$ and $3^{\circ} 54^{\prime} 26^{\prime \prime} \mathrm{N}$, and longitude $8^{\circ} 57^{\prime} 10^{\prime \prime} \mathrm{E}$ and $9^{\circ} 30^{\prime} 49^{\prime \prime} \mathrm{E}$ (Figure 1). Tiko has a land area of $48,400 \mathrm{~km}^{2}$ [19] and a population of 134,649 inhabitants as of 2010. This mangrove zone, at the foot of Mount Cameroon, is located on the Cameroon Atlantic Coast of the West African Gulf of Guinea [20]. It has a maritime to equatorial climate with a short dry season (December to February) and a long rainy season (March to November). The area receives excessive rainfall with mean annual rainfall of 3000 to $4000 \mathrm{~mm}$. Relative humidity ranges from $80 \%$ to $95 \%$ depending on the season while mean annual temperature ranges from $28^{\circ} \mathrm{C}$ to $35^{\circ} \mathrm{C}$. Originally evergreen lowland forest and mangrove swamps, $80 \%$ of the forest land of Tiko municipality has been converted to oil palm, rubber and banana plantations by Cameroon Development Corporation (CDC) and only few patches of secondary forests exist. The creeks harbour large areas of mangrove forests which are very highly exploited for wood. The soils are ferralitic, volcanic and very fertile [21]. The principal activity employing over $75 \%$ of the cosmopolitan population is agriculture. In addition to the CDC plantations, there are also small scale farms producing cocoa and food crops. Other economic activities of the town include trading, fishing and wood extraction. Unfortunately the supply of energy to this dense population is erratic and expensive. Regular power cuts and irregular gas supply together with poverty have pushed the population to depend on biomass. The biomass comes mainly from old rubber trees and mangroves. The high density mangrove wood is preferred for construction poles, fish smoking, charcoal production and firewood for home use.

Sampling sites were identified (Figure 1, Table 1) after a reconnaissance survey in 2012 based on the duration of perturbation in the locality within the mangroves through interviews of locals.

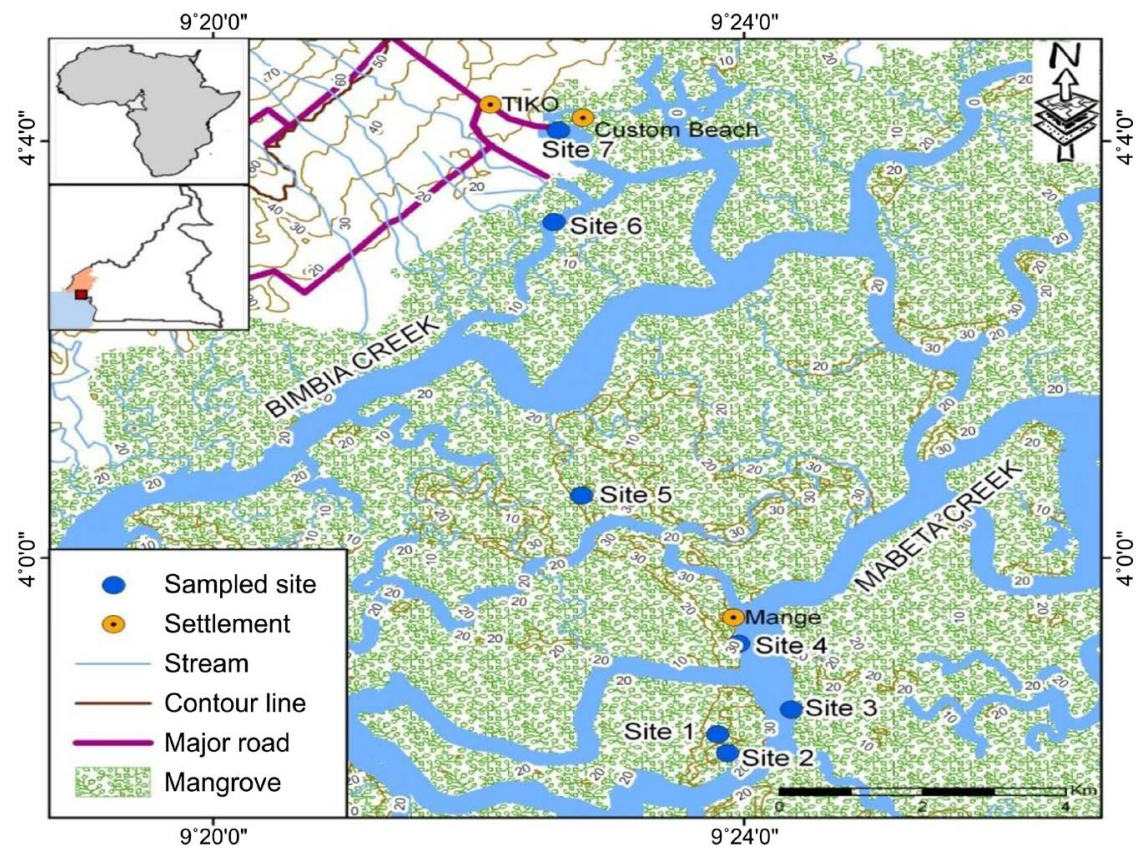

Figure 1. Location of study sites and sampling points within the Tiko mangroves. 
Table 1. Description of the various sampling sites.

\begin{tabular}{|c|c|c|c|}
\hline Site & Location & Elevation $(\mathrm{m})$ & Description \\
\hline 1 & $\begin{array}{l}03^{\circ} 58^{\prime} 18.2^{\prime \prime} \mathrm{N} \\
009^{\circ} 23^{\prime} 47.9^{\prime \prime} \mathrm{E}\end{array}$ & 4 & $\begin{array}{l}\text { New harvesting site. Wood harvesting began just before } \\
\text { survey }\end{array}$ \\
\hline 2 & $\begin{array}{l}03^{\circ} 58^{\prime} 07.6^{\prime \prime} \mathrm{N} \\
009^{\circ} 23^{\prime} 52.3^{\prime \prime} \mathrm{E}\end{array}$ & 4 & Site had been under wood and pole extraction for one year \\
\hline 3 & $\begin{array}{l}03^{\circ} 58^{\prime} 32.5^{\prime \prime} \mathrm{N} \\
009^{\circ} 24^{\prime} 21.3^{\prime \prime} \mathrm{E}\end{array}$ & 4 & Human settlement and fish smoking for about 60 years \\
\hline 4 & $\begin{array}{l}03^{\circ} 59^{\prime} 10.5^{\prime \prime} \mathrm{N} \\
009^{\circ} 23^{\prime} 57.3^{\prime \prime} \mathrm{E}\end{array}$ & 8 & $\begin{array}{l}\text { Wood and pole extraction for about } 20 \text { years with natural } \\
\text { regeneration in progress }\end{array}$ \\
\hline 5 & $\begin{array}{l}04^{\circ} 00^{\prime} 35.8^{\prime \prime} \mathrm{N} \\
009^{\circ} 22^{\prime} 46.4^{\prime \prime} \mathrm{E}\end{array}$ & 10 & Wood and pole extraction for about 30 years \\
\hline 6 & $\begin{array}{l}04^{\circ} 03^{\prime} 13.2^{\prime \prime} \mathrm{N} \\
009^{\circ} 22^{\prime} 34^{\prime \prime} \mathrm{E}\end{array}$ & 10 & $\begin{array}{l}\text { Settlement and small scale agricultural activities. Wood and } \\
\text { pole extraction for about } 40 \text { years }\end{array}$ \\
\hline 7 & $\begin{array}{l}04^{\circ} 04^{\prime} 06.1^{\prime \prime} \mathrm{N} \\
009^{\circ} 22^{\prime} 35.9^{\prime \prime} \mathrm{E}\end{array}$ & 10 & $\begin{array}{l}\text { Settlement with small scale agricultural activities. Wood and } \\
\text { pole extraction for about } 70 \text { years }\end{array}$ \\
\hline
\end{tabular}

\subsection{Assessment of Drivers of Change in the Tiko Mangroves}

Semi-structured questionnaires and open ended interviews were used to assess the different activities in the area as drivers of change that degrade the mangrove wetlands in Tiko. Purposive sampling techniques were used to select the respondents with the questionnaires having been divided into two main groups. The first group of respondents was made up of the general public living in and around the creeks. Here 100 randomly selected respondents answered questions related to their use of products derived from the mangrove ecosystem. The second set of questionnaires targeted professionals such as loggers, wood and pole collectors, wood splitters, charcoal producers, fishermen and fish smokers. Here, 60 questionnaires/interviews were administered. The first part of all questionnaires/interviews collected demographic data while the second part focused on their activities in the mangroves.

\subsection{Sample Collection and Analyses}

Two sets of composite sediment samples in triplicates were collected at low tide from each site. Samples for physico-chemical characterization were collected using a soil corer. At each site, a total of 10 randomly collected core samples were bulked and subsampled and put in labeled plastic bags for transportation. Samples were air dried to constant weight according to Reddy [22], crushed in a mortar and sieved through a $2 \mathrm{~mm}$ sieve. Each sample was subsampled, packaged, coded and sent to the Soil and Environmental Chemistry Laboratory of the University of Dschang for analyses. Particle size was determined using Bouyoucos hydrometer method, while the $\mathrm{pH}$ was measured using a $\mathrm{pH}$ meter with a glass electrode. Available phosphorus was analysed by the Bray II method. Total nitrogen and ammonium were determined by the Kjeldahl's method. The levels 
of the exchangeable bases $\left(\mathrm{Na}^{+}, \mathrm{K}^{+}, \mathrm{Mg}^{2+}\right.$ and $\left.\mathrm{Ca}^{2+}\right)$ were determined using flame spectrophotometry. Organic carbon was analyzed by the Black [23] method. The amount of organic matter was determined by the formula:

$$
\% \mathrm{OM}=\% \mathrm{OC} \times 1.724 \quad[24] \text {. }
$$

Samples for benthic algal analysis were collected and processed according to Danielson [25]. Samples were collected using petri dishes and trowel. Composite core samples were transferred into beaker and distilled water added to the mark of $150 \mathrm{~mL}$. Samples were then poured from the beaker into wide-mouth, nalgene bottles and 3 drops of 10\% Lugol's iodine added to each bottle to fix and preserve cell structure. They were then transported to the Life Sciences laboratory of the University of Buea in ice-filled cooler [26] for analysis. A drop of each centrifuged (2500 rpm for 20 minutes) sediment sample was mounted on a clean slide. Counting and identification of species was done using an Olympus BH - 2 light microscope equipped with Normaski optics at a magnification of $1000 \mathrm{X}$. Slides for quantitative and qualitative analyses were prepared in triplicates, and whole count method was employed. Following counting, extrapolation was done by multiplying the count from three drops $(3 \mathrm{ml}$ ) by a factor of 333.33 to get the number of cells per litre. The Sedgwick Rafter counting chamber was used to determine algal density [10]. Identification of algae followed relevant text books and publications, including [27]-[38] and were classified according to algaebase.org. [39].

\subsection{Data Analysis}

Data obtained from the questionnaires were analyzed using Microsoft Excel 2010 to produce simple charts and other descriptive statistics for each of the parameters in all the questionnaires. Kolmogorov-Smirnov test for normality at $\alpha$ $=0.05$ showed that sediment nutrient data were normally distributed and suitable for parametric analyses (ANOVA) while species distribution and abundance data was not. As a result, distribution and abundance of species across sites was compared using the Kruskal-Wallis non-parametric ANOVA. For distribution, presence/absence data was used, with presence coded as $=1$ and absence of species $=0$. Simple Correspondence Analyses were then done, to investigate spatial associations of the species distribution and abundance within the different sites and nutrient distribution across sites. To assess diversity and similarity of the different sites, the following indices were calculated:

\subsubsection{Shannon Diversity $\left(H^{\prime}\right)$ : Species Diversity

$$
H^{\prime}=\sum\left(P_{i}\right)\left(\log _{n} P_{i}\right)
$$

where:

$H^{\prime}=$ Index of species diversity (information content of sample, bits or individuals)

$P_{i}=$ Proportion of total sample belonging to $i$ th species

$i=$ Number of species. 


\subsubsection{Margalef Index $(d)$ : Species Richness}

$$
d=(S-1) \log _{e} N
$$

where:

$$
\begin{aligned}
& d=\text { Species richness } \\
& S=\text { number of species identified } \\
& N=\text { total number of individuals }
\end{aligned}
$$

\subsubsection{Pielou Index: Species Evenness}

$$
J^{\prime}=\frac{H^{\prime}}{-\sum_{i-1}^{s} \frac{1}{S} \ln \left(\frac{1}{S}\right)}=\frac{H^{\prime}}{\ln (S)}
$$

where:

$J^{\prime}$ is species evenness, $H^{\prime}$ is the observed Shannon's index, and $S$ is the species richness.

\subsubsection{Sorenson' Similarity Index}

$$
C n=2 C / A+B
$$

where:

$C n=$ Sorensen's similarity coefficient

$C=$ number of species common to both sites

$A$ and $B=$ number of species in each site

Pearson correlations were also conducted for sediment nutrients and benthic community descriptors, and general correlation of all variables. All analyses were done at $\alpha=0.05$, using the Minitab Version 16 statistical package (Minitab Inc., USA).

\section{Results}

\subsection{Drivers of Change around the Tiko Mangrove Swamp}

Seven different activities or drivers of change were identified namely agriculture, human settlement, logging, fuel wood collection, fishing, fish smoking and petty trading (Figure 2).

More than half of the population (52.6\%) use mangroves as fuel wood, $27.6 \%$ use it for smoking fish, $13.2 \%$ use it for building, while $2.6 \%$ use the mangrove plants to make charcoal and $5.3 \%$ as props during the construction of storey buildings (Figure 3). Rhizophora mangle (red mangrove) was the most used species.

The perception of respondents on ecological changes in the Tiko mangrove swamps showed that $68.4 \%$ noticed changes in tree size, with the consequence that loggers have to move further into the creek to meet reasonable size of trees to cut. Some respondents reported an increase in water temperature (2.6\%) and observed soil erosion in the mangrove swamps (18.5), while others noticed a reduction in water volume (5.3\%). They reported a general drop in the quantity and quality of fish $(22.4 \%)$ caught, as fisher men have to move further into the 


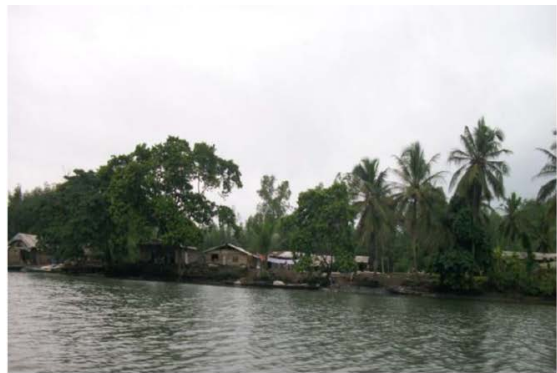

Settlement area

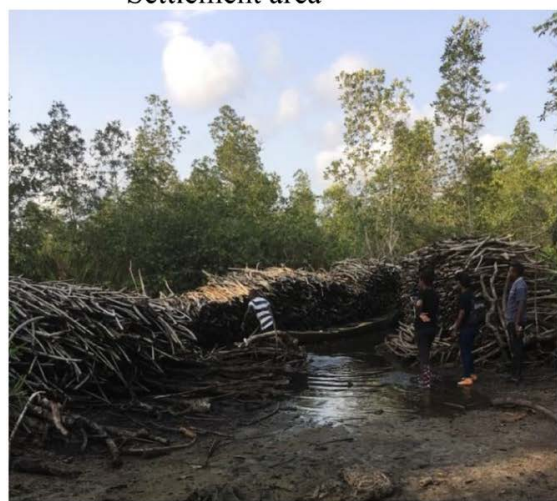

Mangrove wood stocked

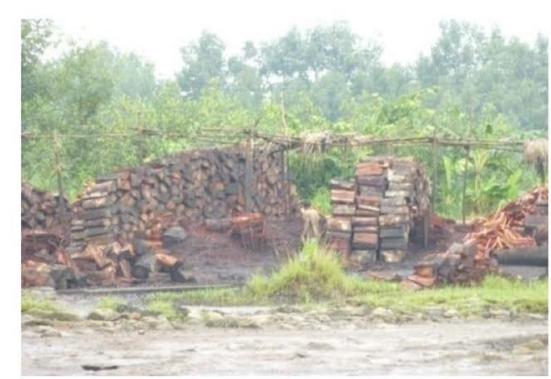

Wood splitting and transportation

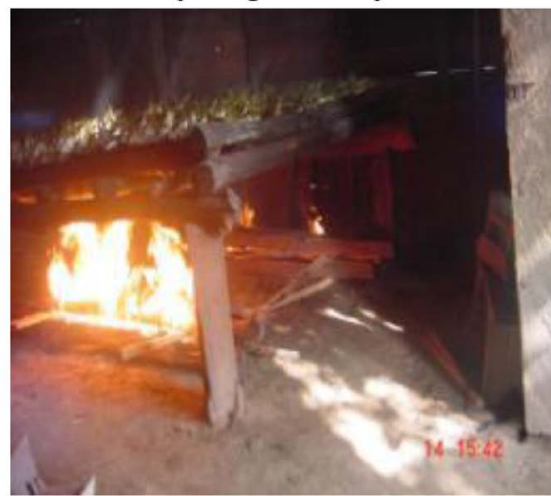

Fish smoking

Figure 2. Some drivers of change in the Tiko mangrove estuary.

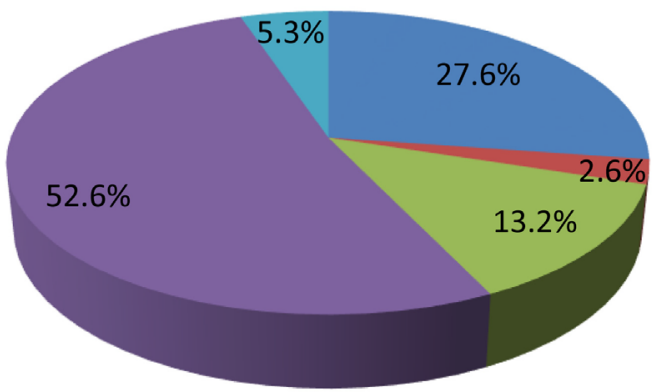

Mangrove use

Fish smoking

- Chacoal making

Building

Fuel wood

- Props

Figure 3. Main uses of mangroves by people associated to Tiko Mangrove swamps.

creeks. Some respondents $(31.6 \%)$ did not notice any changes in the mangrove swamps.

\subsection{Variations in Physicochemical Characteristics of Sediments}

The properties of the sediments from the different sites varied as indicated in Table 2.

Sediment texture was dominated by clay followed by silt in all the sites. Sediment $\mathrm{pH}$ in KCL and water were generally low, ranging from 2.9 to 5.0, an indication of acidic conditions.

As expected, both organic carbon and organic matter levels were very high across all sites. In the current study, only site 3 (settlements and small scale agriculture) had organic carbon value of 4.82. While nutrient levels were generally moderate to high across sites, $\mathrm{Mg}$ values were particularly high for most of 
Table 2. Physicochemical characteristic of sediments at different mangrove sites.

\begin{tabular}{cccccccc}
\hline Parameters & Site 1 & Site 2 & Site 3 & Site 4 & Site 5 & Site 6 & Site 7 \\
\hline Sand (\%) & 14 & 32 & 31 & 32 & 24 & 19 & 30 \\
Silt (\%) & 17 & 24 & 27 & 37 & 17 & 14 & 23 \\
Clay (\%) & 69 & 44 & 42 & 31 & 59 & 67 & 47 \\
pH H2O & 3.7 & 4.9 & 4.3 & 4.3 & 5.0 & 4.7 & 3.0 \\
pH KCL & 3.3 & 4.1 & 4.0 & 4.0 & 4.3 & 4.2 & 2.9 \\
Org Carb (\%) & 4.75 & 4.81 & 2.81 & 4.62 & 4.55 & 4.42 & 4.94 \\
Org Mat (\%) & 8.18 & 8.29 & 4.84 & 7.96 & 7.85 & 7.63 & 8.52 \\
Al (ppm) & 0.09 & 0.22 & 0.13 & 0.18 & 0.09 & 0.09 & 0.18 \\
Avail P (ppm) & 2.65 & 3.44 & 2.61 & 2.52 & 2.32 & 11.4 & 8.13 \\
CEC (meq/100g) & 25.52 & 29.28 & 15.68 & 24.4 & 17.6 & 27.2 & 22.88 \\
N-NO $(\%)$ & 1.83 & 0.98 & 0.27 & 1.25 & 1.24 & 0.82 & 0.46 \\
Total N (\%) & 0.43 & 0.23 & 0.06 & 0.29 & 0.29 & 0.19 & 0.11 \\
$\mathrm{~N}-\mathrm{NH} \mathrm{H}_{4}^{+}(\%)$ & 0.55 & 0.3 & 0.08 & 0.38 & 0.37 & 0.25 & 0.14 \\
$\mathrm{Ca}(\mathrm{meq} / 100 \mathrm{~g})$ & 3.04 & 8.08 & 4.88 & 2.48 & 7.28 & 2.48 & 2.72 \\
$\mathrm{Mg}(\mathrm{meq} / 100 \mathrm{~g})$ & 43.12 & 54.56 & 4.72 & 57.32 & 16.96 & 31.1 & 19.68 \\
$\mathrm{~K}(\mathrm{meg} / 100 \mathrm{~g})$ & 2.01 & 1.67 & 0.47 & 0.86 & 1.84 & 1.37 & 0.32 \\
$\mathrm{Na}(\mathrm{meq} / 100 \mathrm{~g})$ & 3.83 & 3.34 & 1.09 & 3.34 & 3.34 & 1.7 & 0.62 \\
$\mathrm{Base} \mathrm{sat} \mathrm{(meq/100g)}$ & 5166 & 6188 & 21509 & 12016 & 5616 & 7423 & 31444 \\
$\mathrm{ECEC}(\mathrm{cmol} / \mathrm{Kg})$ & 103.83 & 103.34 & 101.09 & 103.34 & 103.34 & 101.7 & 100.62 \\
\hline
\end{tabular}

${ }^{\star}$ Effective Cation Exchange Capacity.

the sites. $\mathrm{Mg}$ concentration at Site 4 was $57.32 \mathrm{meq} / 100 \mathrm{~g}$ while at Site 3 it was $4.72 \mathrm{meq} / 100 \mathrm{~g}$. In this study ECEC was high in all the sites with values $\geq 100.62$ $\mathrm{cmol} / \mathrm{Kg}$. The values of the major cations ( $\mathrm{Ca}, \mathrm{Mg}, \mathrm{Na}$ and $\mathrm{K}$ ) showed a lot of variations in the soils in this study across sites. Ca levels ranged from 2.48 to 8.08 meq/100g.

\subsection{Benthic Algal Community of the Tiko Mangroves}

A total of 141 algal species belonging to 53 families and 10 divisions were identified in all study sites. The division with the highest number of species was the Bacillariophyta (60 species) followed by Chlorophyta (38 species), Cyanophyta (16 species) and Euglenophyta (8 species) (Table 3).

The most abundant family was the Fragilariaceae with 14 species, while 32 families were represented by only one species each (Table 4). The most abundant species were Microcytis sp $(24.09 \%$ at Site $7,18.5 \%$ at Site 4 , and $15.6 \%$ at Site 6), Chlorobotrys regularis (24.5\% at Site $1,17.1 \%$ at Site 5 and $14.8 \%$ at Site 3), Microceleus sp (12.9\% at Site 5), Synedraacus var radians (18.5\% at Site 2) and Tribonema sp (18.5\%). 
Table 3. Benthic Division and the number of species across the study sites.

\begin{tabular}{ccccccccc}
\hline & \multicolumn{7}{c}{ Number of species } \\
\hline Division & No of species & Site 1 & Site 2 & Site 3 & Site 4 & Site 5 & Site 6 & Site 7 \\
\hline Bacillariophyta & 61 & 30 & 19 & 33 & 33 & 30 & 19 & 14 \\
Chlorophyta & 38 & 16 & 17 & 20 & 15 & 19 & 14 & 9 \\
Cryptophyta & 2 & 2 & 1 & 2 & 1 & 1 & 1 & 0 \\
Cyanophyta & 16 & 12 & 7 & 9 & 8 & 11 & 7 & 3 \\
Dinophyta & 8 & 3 & 4 & 5 & 5 & 3 & 1 & 1 \\
Euglenophyta & 8 & 2 & 6 & 1 & 4 & 1 & 0 & 2 \\
Eustigmatophyta & 1 & 1 & 0 & 1 & 1 & 1 & 1 & 1 \\
Phaeophyta & 2 & 0 & 0 & 0 & 1 & 0 & 0 & 1 \\
Rhodophyta & 1 & 0 & 0 & 0 & 1 & 0 & 0 & 0 \\
Xanthophyta & 4 & 0 & 1 & 1 & 2 & 3 & 1 & 2 \\
Total & 141 & $\mathbf{6 6}$ & $\mathbf{5 5}$ & $\mathbf{7 2}$ & $\mathbf{7 1}$ & $\mathbf{6 9}$ & $\mathbf{4 4}$ & 33 \\
\hline
\end{tabular}

Table 4. Checklist of Benthic algal species in the Tiko mangrove swamps.

\begin{tabular}{|c|c|c|}
\hline Division & Family & Species \\
\hline \multirow{22}{*}{ Bacillariophyta } & & Achnanthes adamantiformis \\
\hline & Achnanthaceae & Achnanthes hungarica \\
\hline & & Nitzschia capitellata \\
\hline & & Nitzschia flexoides \\
\hline & Bacillariaceae & Nitzschia intermedia \\
\hline & & Nitzschia ignorata \\
\hline & & Nitzschia periata \\
\hline & Biddulphiaceae & Biddulphia mobiliensis \\
\hline & & Coscinodiscus alborani \\
\hline & Coscinodiscaceae & Coscinodiscus furcatus \\
\hline & & Coscinodiscus radiatus \\
\hline & Diplonieceae & Diplopsalis sp \\
\hline & Eupodiscaceae & Biddulphia sinensis \\
\hline & \multirow{9}{*}{ Fragilariaceae } & Asterionella sp \\
\hline & & Diatoma elongatum \\
\hline & & Diatoma triemale \\
\hline & & Fragilaria acus \\
\hline & & Fragilaria brevistriata \\
\hline & & Fragelaria construens var javanica \\
\hline & & Fragilaria capucina \\
\hline & & Fragilaria crotonensis \\
\hline & & Fragilaria heidenii \\
\hline
\end{tabular}




\section{Continued}

Fragilaria pinnata

Fragilaria virescens

Fragilaria $s p$

Synedra acus var radians

Asterionella japonica

Diatoma vulgare

\begin{tabular}{cc}
\hline Gomphonemataceae & Gomphonema sp \\
\hline Leptocylindraceae & Leptocylindricus danicus \\
\hline Licmophoraceae & Licmophora sp \\
\hline Mastogloiaceae & Mastogloia baltica \\
\hline & Navicula buderii \\
Gyrosigma $s p$
\end{tabular}

Gyrosigma spencerii

Navicula complanata

Navicula $s p$

Navculaceae

Navicula $s p 2$

Nitzschia dissipata

Nitzschia frustulum var bunheimiana

Nitzschia glacici van Hauck

Nitzschia latens

Nitzschia subodiosa

Gyrosigma attenuatum

Pleurosigmataceae

Pleurosigma directum

Pleurosigma normanii

Rhopalodia acuminata

Rhopalodiaceae

Rhopalodia gibba

Rhopalodia gibberula var magnusii

Cyclotella glomerata

Cyclotella bodanica

Cyclotella stelligera

Amphora coffeaeformis

$\begin{array}{ll}\text { Amphora coffeaeformis } \\ \text { Surirellaceae } & \text { Campylodiscus hibernicus }\end{array}$

Surirella spiralis

Tabellariaceae

Tabellaria $s p$

Tabellaria flocculosa

Thalassiosira lentignosa

Thalassionemataceae

Thalassiothrix longissima

Trichotoxon reinboldii

Bacillaria paxillifera

Chlorophyta

Bacillariaceae

Pleurotaenium cylindrium 


\section{Continued}

\begin{tabular}{cc} 
& Pleurotaenium excelsum \\
\hline Chlamydomonadaceae & Chlamydomonas penium \\
\hline Chlorellaceae & Chlorella $s p$ \\
\hline \multirow{2}{*}{ Chlorococcaceae } & Characium $s p$ \\
& Closterium $s p$ \\
\hline Chlorophyceae & Palmodictyon $s p$ \\
\hline \multirow{2}{*}{ Cladophoraceae } & Cladophora $s p 1$ \\
& Cladophora $s p 2$ \\
\hline & Cosmarium $s p$
\end{tabular}

Closterium abruptum

Closterium parvulum

Closterium arcuarium

Desmidaceae

Closterium kuetzingii f minus

Cosmarium clepsydra var dissimile

Cosmarium monilliformis

Spenoclosterium curoatum

Closterium exile

Euastropsis $s p$

Hydrodictyaceae

Hydrodictyon $s p$

Tetraedron $s p$

\begin{tabular}{ll} 
& Tetraedron $s p$ \\
\hline Microsporaceae & Microspora $s p$ \\
\hline
\end{tabular}

\begin{tabular}{cc}
\hline Nostocaceae & $\begin{array}{c}\text { Gloeocystis plantonica } \\
\text { Raphidiopsis } s p\end{array}$ \\
\hline Oocystaceae & Oocystis solilaria \\
Palmellaceae & Palmella $s p$ \\
Prasinophyceae & Resultoe mikron \\
\hline Scenedesmaceae & Actimastrum $s p$ \\
& Scenedesmus $s p$
\end{tabular}

Scenedesmus obliquus

\begin{tabular}{cc} 
& Scenedesmus obliquus \\
\hline Selanastraceae & Ankistrodesmus $s p$ \\
Sphaeropleaceae & Sphaeroplea $s p$
\end{tabular}

Thalassionemataceae

Thalassionema mitzchroides

Trebouxiophyceae

Trebouxia sp

Ulotrichaceae Stichococcus sp

\begin{tabular}{rr} 
Ulotrichaceae & Stichococcus $s p$ \\
\hline Zygnemataceae & Zygnema $s p$ \\
& Pleurodiscus boring
\end{tabular}

Cryptophyta

Cryptomonadaceae

Cryptomonas $s p$

Pyrenomodaceae

Rhodomonas $s p$ 
G. A. Neba et al.

\section{Continued}

\begin{tabular}{|c|c|c|}
\hline \multirow{16}{*}{ Cyanophyta } & Cyanophyceae & Dactylococcopsis $s p$ \\
\hline & \multirow{4}{*}{ Microcystaceae } & Anacystic sp1 \\
\hline & & Anacystic sp2 \\
\hline & & Anacystic sp3 \\
\hline & & Microcystis sp \\
\hline & \multirow{5}{*}{ Oscillatoriaceae } & Chlorobacterium aggregatum \\
\hline & & Lyngbya birger \\
\hline & & Microceleus sp \\
\hline & & Oscillatoria $s p$ \\
\hline & & Oscillatoria brevis \\
\hline & Phormidiaceae & Phormidium autumale \\
\hline & Rivulariaceae & Calothrix $s p$ \\
\hline & Scytonemataceae & Scytonema arcangelii \\
\hline & \multirow{3}{*}{ Surirellaceae } & Stenopterobia ancepts \\
\hline & & Stenopterobia curvula \\
\hline & & Stenopterobia $s p$ \\
\hline \multirow{5}{*}{ Dinophyta } & Chrysocapsaceae & Dinobryon balticum \\
\hline & \multirow{2}{*}{ Dinophysiaceae } & Dinophysis acuminoua \\
\hline & & Dinophysis sp \\
\hline & Peridiniaceae & Peridinium breve \\
\hline & Warnowiacea & Erythropsis pavillandi \\
\hline \multirow{7}{*}{ Euglenophyta } & \multirow{6}{*}{ Euglenaceae } & Euglena $s p$ \\
\hline & & Phacus sp \\
\hline & & Trachelomonas volzii \\
\hline & & Trachelomonas conica f punctata \\
\hline & & Trachelomonas olonga \\
\hline & & Trachelomonas $s p$ \\
\hline & Vaucheriaceae & Vaucheria \\
\hline Eustigmatophyta & Chlorobothryaceae & Chlorobotrys regularis \\
\hline \multirow{2}{*}{ Phaeophyta } & \multirow{2}{*}{ Gephyrocapsaceae } & Phaeocystis globosa \\
\hline & & Phaeocystis sp \\
\hline \multirow{3}{*}{ Pyrrophycophyta } & \multirow{3}{*}{ Prorocentraceae } & Cenchridium globosum \\
\hline & & Cenchridium sphaerula \\
\hline & & Prorocentrum minimum \\
\hline \multirow[t]{2}{*}{ Rhodophyta } & Porphyridiaceae & Chroodactylon $s p$ \\
\hline & & Goniochloris $s p$ \\
\hline \multirow[t]{4}{*}{ Xanthophyta } & Gloeobotrydaceae & Goniochloris sp2 \\
\hline & & Goniochloris sp3 \\
\hline & Tribonemataceae & Tribonema $s p$ \\
\hline & Epithemiaceae & Epithemia sp \\
\hline
\end{tabular}


The mean abundance of benthic algae ranged from $0.59 \pm 0.17$ to $2.08 \pm 0.58$. Site 4 had the highest mean benthic algal abundance while Site 6 had the least (Figure 4).

The Kruskal-Wallis test comparing abundance across sites showed there exist significant differences between different sites $(\mathrm{H}=42.52, \mathrm{DF}=6, \mathrm{P}<0.01)$ and the following groups showed significant differences; Sites 3 and $7(Z=4.93, P<$ $0.001)$, Sites 5 and $7(Z=4.54, P<0.001)$, Sites 4 and $7(Z=4.35, P<0.001)$, Sites 3 and $6(Z=3.93, P<0.0001)$, Sites 5 and $6(Z=3.55, P=0.0004)$, and Sites 4 and $6(Z=3.35, P=0.0008)$.

Observed patterns of species abundance across sites were explained by two main components. The first two components explained $29.62 \%$ and $20.72 \%$ (Table 5) of the total abundance of species. These first two components cumulatively contributed $50.34 \%$ of the total inertia. The two components effectively explain species abundance across all sites (Qual $=0.977$ and 0.026 respectively). Component 1 explained abundance of species at Site 2 (Corr $=0.896)$. Synedra acus var radians, Trachelomonas conica f punctata, Resultoe mikron, Fragilaria capucina, Epithemia sp and Closterium parvulum have highest abundance at Site 2 , and occur at very low numbers in other sites. Sites 5 and 7 were best explained by Component 2 (corr $=0.533$ and 0.283 respectively). Coscinodiscus alborani (10), Coscinodiscus radiatus (11), Microceleus sp (38), Palmella sp (18), Tabellaria sp, Gyrosigma spencerii, Goniochloris sp, and Biddulphia mobiliensis, have their highest abundance at Site 5. Similarly, Phaeocystis globosa, and Rhopalodia acuminate (10) have the highest abundance at Site 7, occurring in very low numbers across the other sites (Table 5, Figure 5).

Table 5. Correspondence Analysis of benthic species abundance across sites.

\begin{tabular}{|c|c|c|c|c|c|c|c|}
\hline \multicolumn{8}{|c|}{ Analysis of Contingency table } \\
\hline Axis & 1 & 2 & 3 & 4 & 5 & 6 & Total \\
\hline Inertia & 0.4333 & 0.3032 & 0.2111 & 0.1904 & 0.1727 & 0.1525 & 1.4632 \\
\hline Proportion & 0.2962 & 0.2072 & 0.1443 & 0.1302 & 0.118 & 0.1042 & \\
\hline Cumulative & 0.2962 & 0.5033 & 0.6476 & 0.7778 & 0.8958 & 1 & \\
\hline \multicolumn{8}{|c|}{ Row contribution } \\
\hline & \multicolumn{4}{|c|}{ Component 1} & \multicolumn{2}{|c|}{ Component 2} & \\
\hline Name & Qual & & Corr & Contr & Corr & Contr & \\
\hline Site 1 & 0.026 & & 0.001 & 0 & 0.025 & 0.013 & \\
\hline Site 2 & 0.977 & & 0.896 & 0.72 & 0.082 & 0.094 & \\
\hline Site 3 & 0.044 & & 0.008 & 0.003 & 0.036 & 0.016 & \\
\hline Site 4 & 0.263 & & 0.027 & 0.011 & 0.236 & 0.136 & \\
\hline Site 5 & 0.968 & & 0.435 & 0.255 & 0.533 & 0.447 & \\
\hline Site 6 & 0.193 & & 0.023 & 0.011 & 0.17 & 0.112 & \\
\hline Site 7 & 0.284 & & 0.001 & 0 & 0.283 & 0.182 & \\
\hline
\end{tabular}

Qual = the proportion of the row inertia represented by the two components. Coord = the principal coordinates of the rows. Corr $r=$ the contribution of the component to the inertia of the row. Contr $=$ the contribution of each row to the axis inertia, Inert = the proportion of the total inertia contributed by each row. 


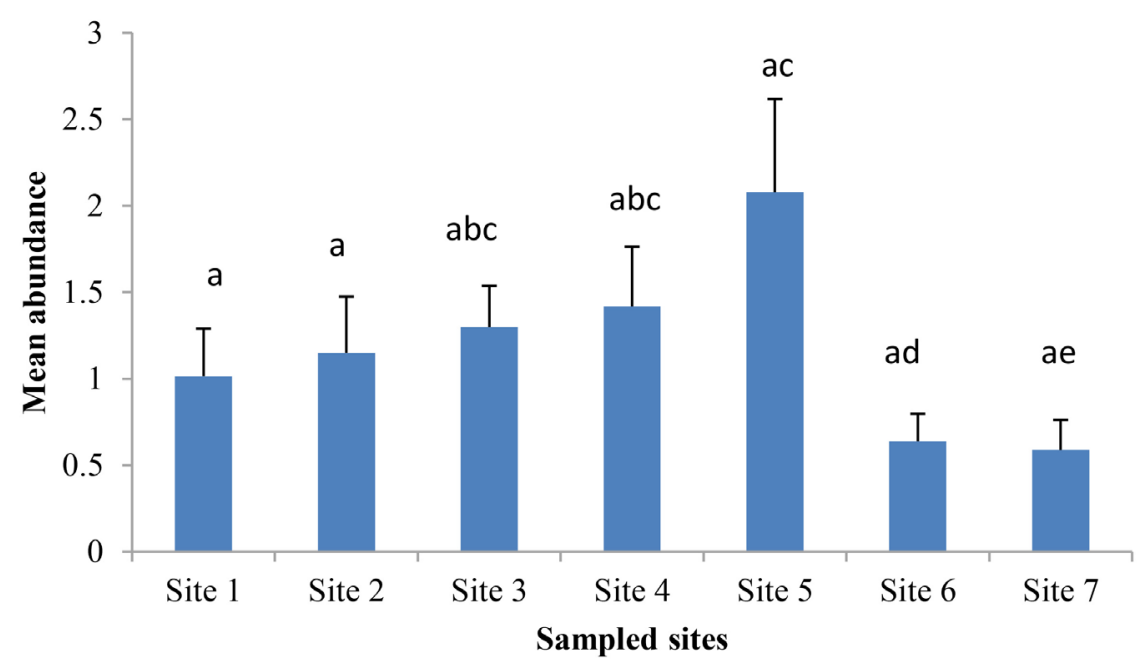

Figure 4. Mean Abundance of Benthic algal species in the different study sites. Bars represent means $\pm \mathrm{SE}$, means separated using Kruskal-Wallis ANOVA with multiple comparison bars with the same letter are not significantly different.

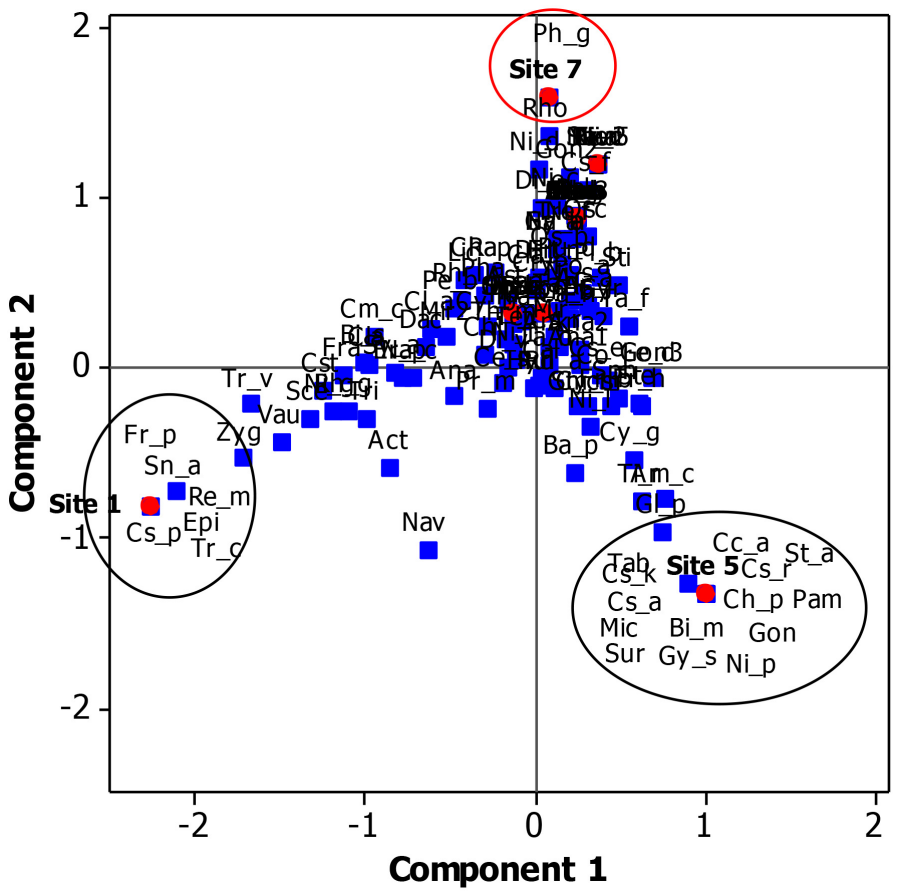

Figure 5. Species association of benthic abundance across sites. Red dot $=$ sites, Blue dots $=$ Benthic species. Biddulphia mobiliensis $=$ Bi_m, Closterium abruptum $=C s \_a$, Closterium parvulum $=C s_{-} p$, Closterium arcuarium $=C s_{-} r$, Closterium kuetzingii $f$ minus $=$ $C s \_k$, Coscinodiscus alborani $=C c_{\_} a$, Coscinodiscus radiates $=C s \_r$, Chlamydomonas penium $=C h \_p$, Epithemia $s p=E p i$, Fragilaria capucina $=F r_{-} p$, Goniochloris $s p=$ Gon, Gyrosigma spencerii $=G_{-} s$, Microceleus $s p=$ Mic, Nitzschia periata $=N i \_$, Palmella sp $=$ Pam, Phaeocystis globosa $=$ Ph_g, Resultoe micron $=R e \_m$, Rhopalodia acuminate $=$ Rh_a, Surirella spiralis $=$ Sur, Synedra acus var radians $=S n \_a$, Tabellaria $s p=T a b$, Trachelomonas conica $f$ punctata $=T r_{-} c$, Actimastrum $=A c t$, Zygnema sp $=Z y g, C o s m a-$ rium clepsydra var dissimile $=C m_{-} c$, Cyclotella glomerata $=C y_{-} g$, Navicula sp $=N a v$, Bacillaria paxillifera $=$ Ba_p, Trachelomonas volzii $=T r_{-} v$, Vaucheria $=$ Vau, Prorocentrum minimum $=P r_{-} m$, Gloeocystis plantonica $=G I \_$, Amphora coffeaeformis $=$Am_c. 


\subsection{Diversity, Evenness, Richness, Mean abundance and Euglenophycean Index of Benthic Species in Tiko Mangroves}

Site 3 was found to be the most diverse $\left(H^{\prime}=3.81\right)$ while Site 7 was the least diverse $\left(H^{\prime}=2.943\right)$. The most abundant species were recorded at Site $5(4.246)$ and the least at Site 6 (2.045). Seventy two (72) species were recorded at Site 3, and the lowest number of species (33) was recorded at Site 7 (Table 6). Species evenness varied across sites, with site 3 being the most even (0.8909) while site 5 was the least (0.7822), an indication that site 5 had a few dominant species. The highest value of the Euglenophycean index was recorded in site $2(0.21)$, while the lowest was in site $6(0)$ as none of the species used in the calculation was present.

Several sites recorded a number of common species (Table 7), an indication of similar biophysical conditions. Sites 3 and 5 shared the highest number of common species (41) while Sites 6 and 7 had the least number (3).

Similarities between the sites are presented in Table 8 . It was seen here that Sites 1 and 2 were very similar with their high value of 0.606 . Sites 6 and 7 were the least similar (0.338). Despite their close proximity and similar activities, these sites vary in physicochemical properties as site 7 receives both industrial and urban effluents.

Table 6. Descriptors of Benthic community in the mangrove wetland of Tiko.

\begin{tabular}{cccccc}
\hline Sampling site & $\begin{array}{c}\text { Benthic } \\
\text { Diversity }\end{array}$ & $\begin{array}{c}\text { Benthic } \\
\text { Evenness }\end{array}$ & $\begin{array}{c}\text { Benthic } \\
\text { abundance }\end{array}$ & $\begin{array}{c}\text { Benthic } \\
\text { Richness }\end{array}$ & $\begin{array}{c}\text { Euglenophycean } \\
\text { index }\end{array}$ \\
\hline Site 1 & 3.343 & 0.8380 & 2.648 & 54 & 0.07 \\
Site 2 & 3.166 & 0.7901 & 2.945 & 55 & 0.21 \\
Site 3 & 3.810 & 0.8909 & 2.542 & 72 & 0.03 \\
Site 4 & 3.506 & 0.8225 & 2.817 & 71 & 0.17 \\
Site 5 & 3.312 & 0.7822 & 4.246 & 69 & 0.03 \\
Site 6 & 3.269 & 0.8639 & 2.045 & 44 & 0 \\
Site 7 & 2.943 & 0.8416 & 2.515 & 33 & 0.17 \\
\hline
\end{tabular}

Table 7. Number of Benthic species common to different sites.

\begin{tabular}{lcccccc}
\hline & Site 1 & Site 2 & Site 3 & Site 4 & Site 5 & Site 6 \\
\hline Site 1 & & & & & & \\
Site 2 & $33^{*}$ & & & & \\
Site 3 & 32 & 35 & & & & \\
Site 4 & 28 & 32 & 39 & & & \\
Site 5 & 25 & 30 & 41 & 38 & & \\
Site 6 & 18 & 24 & 26 & 23 & 27 & \\
Site 7 & 18 & 20 & 25 & 21 & 20 & 13 \\
\hline
\end{tabular}

${ }^{\star}$ Each value in the cell represents species which are common between two sites. 
Table 8. Sorenson similarity indices of benthic algae across the different sites.

\begin{tabular}{lllllll}
\hline & Site 1 & Site 2 & Site 3 & Site 4 & Site 5 & Site 6 \\
\hline Site 1 & & & & & & \\
Site 2 & 0.606 & & & & \\
Site 3 & 0.504 & 0.556 & & & & \\
Site 4 & 0.444 & 0.512 & 0.545 & & & \\
Site 5 & 0.403 & 0.488 & 0.582 & 0.543 & & \\
Site 6 & 0.364 & 0.490 & 0.448 & 0.400 & 0.478 & \\
Site 7 & 0.409 & 0.460 & 0.476 & 0.404 & 0.392 & 0.338 \\
\hline
\end{tabular}

${ }^{\star}$ Each value in the cell represents similarity between two sites. Sorenson range: $0-1$, and sites are more similar as index approaches 1 .

\subsection{Mean Distribution of Benthic Algae across the Different Study Sites}

The mean benthic algal distribution had a ranged from $0.23 \pm 0.04$ to $0.51 \pm$ 0.04 . Benthic algae were more widely distributed at Site 3 and least at Site 7 (Figure 6).

The Kruskal-Wallis test showed significant differences in the distribution of benthic species between sites $(H=38.95, D F=6$ and $P<0.001)$. Site 1 was only significantly different from Site $7(Z=4.73, P<0.0001)$, Site 5 was significantly different from Sites 6 and $7(Z=3.03, P=0.0024$ and $Z=4.37, P=0.0007$ respectively). The following pairs of Sites also showed significant differences. Site 3 and Site 7, Site 4 and Site 7, Site 3 and Site $6(P=0.0007)$, Site 4 and Site $6(P=$ 0.0011), Site 2 and Site $7(P=0.0076)$.

Simple Correspondence analyses showed that the observed benthic species distribution can be explained by two main components. The contingency table indicated that Components 1 and 2 contributed $22.01 \%$ and $20.08 \%$ respectively of the total inertia (Table 9). The cumulative contribution of these two components was $42.09 \%$. The two components effectively explain the pattern of distribution of species across all sites ( $Q u a l=0.036$ and 0.792). Component 2 best explains distribution of species at Site $4($ Corr $=0.79)$ and Site $6($ Corr $=0.341)$. Site 4 was highly associated with Achnanthes hungarica, Cenchridium globosum, Chroodactylon sp, Cosmarium sp, Fragilaria virescens, Dinophysis acuminoua, Euglena sp, Gomphonema sp, Mastogloia baltica, Phacus sp and Trachelomonas olonga which were not found in the other sites. Pleurotaenium cylindrium, Nitzschia intermedia, Nitzschia ignorata, Navicula sp2 and Euastropsis sp were found solely at Site 6 . The distribution of species at Site 2 and Site 5 are best explained by Component 1 (Corr $=0.499$ and 0.696 respectively) (Table 9). Palmella sp, Surirella spiralis, Stenopterobia ancepts, Nitzschia periata, Gyrosigma sp, Coscinodiscus alborani, Closterium kuetzingii f minus, Closterium kuetzingii f minus, Closterium arcuarium, Chlamydomonas penium, and Biddulphia mobiliensis were only found at Site 5. Similarly, Closterium parvulum, Epithemia sp, Fragilaria capucina, Resultoe micron and Trachelomonas conica $f$ 


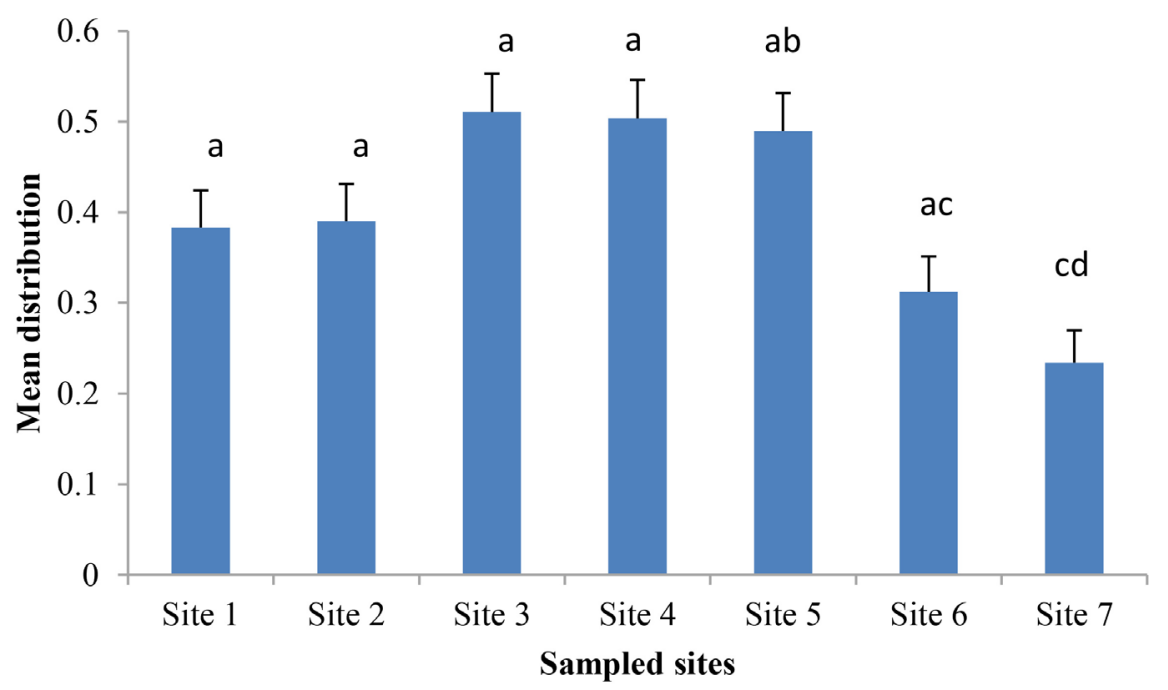

Figure 6. Mean species distribution of Benthic algal across the study sites. Bars represent means \pm SE, means separated using Kruskal-Wallis ANOVA with multiple comparison bars with the same letter are not significantly different.

Table 9. Simple correspondence analysis of species occurrence across sites.

\begin{tabular}{cccccccc}
\hline \multicolumn{7}{c}{ Analysis of Contingency table } \\
\hline Axis & 1 & 2 & 3 & 4 & 5 & 6 & Total \\
\hline Inertia & 0.3113 & 0.284 & 0.2606 & 0.2255 & 0.1842 & 0.1487 & 1.4143 \\
\hline Proportion & 0.2201 & 0.2008 & 0.1843 & 0.1594 & 0.1302 & 0.1051 & \\
Cumulative & 0.2201 & 0.4209 & 0.6052 & 0.7646 & 0.8949 & 1 & \\
\hline & & Row contribution & & & \\
\hline Name & Qual & Corr & Contr & Corr & Contr & & \\
Site 1 & 0.195 & 0.149 & 0.069 & 0.045 & 0.023 & & \\
Site 2 & 0.570 & 0.499 & 0.343 & 0.071 & 0.053 & & \\
Site 3 & 0.004 & 0.001 & 0.000 & 0.003 & 0.002 & & \\
Site 4 & 0.792 & 0.003 & 0.002 & 0.79 & 0.632 & & \\
Site 5 & 0.700 & 0.696 & 0.529 & 0.004 & 0.004 & \\
Site 6 & 0.388 & 0.047 & 0.036 & 0.341 & 0.285 & \\
Site 7 & 0.036 & 0.035 & 0.021 & 0.001 & 0.001 & \\
\hline
\end{tabular}

Qual = the proportion of the row inertia represented by the two components. Coord $=$ the principal coordinates of the rows. Corr $=$ the contribution of the component to the inertia of the row. Contr = the contribution of each row to the axis inertia, Inert = the proportion of the total inertia contributed by each row.

punctata were present only in Site 2 and the rest of species observed were cosmopolitan across sites. Figure 7 shows how the species were associated with the different sites.

Pearson correlations of the different sediment (soil) parameters and benthic community diversity descriptors showed a strong positive correlation between 


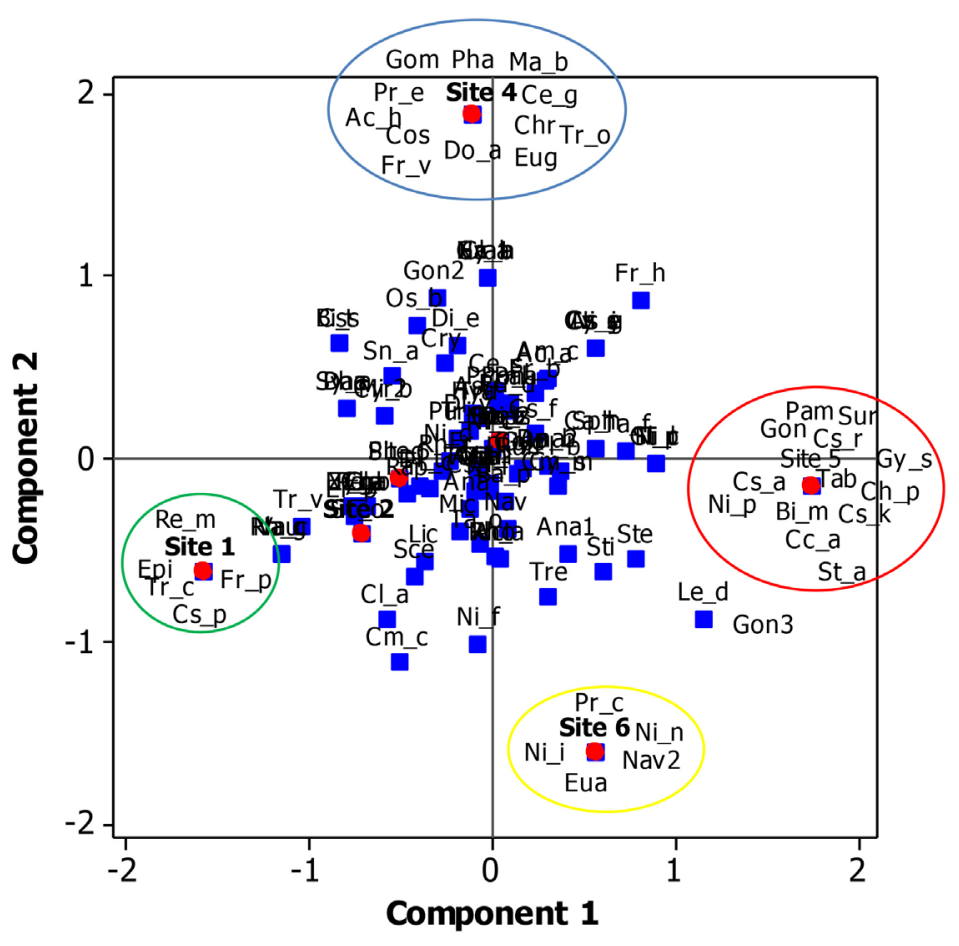

Figure 7. Distribution of benthic species with respect to the different sites. Red dots = sites, Blue dots = benthic species. Achnanthes hungarica $=A c \_h$, Biddulphia mobiliensis $=$ Bi_m, Cenchridium globosum $=C e_{-}$, Chlamydomonas penium $=C h \_p$, Chroodactylon $\mathrm{sp}=C h r$, Closterium abruptum $=C s_{-}$, Closterium parvulum $=C s \_p$, Closterium arcuarium $=C s_{\_} r$, Closterium kuetzingii $f$ minus $=C s_{\_} k$, Coscinodiscus alborani $=C c_{-} a$, Coscinodiscus radiates $=C s_{-} r$, Dinophysis acuminoua $=$ Do_a, Epithemia $s p=E p i$, Euastropsis $\mathrm{sp}=$ Eua, Euglena $\mathrm{sp}=$ Eug, Fragilaria capucina $=F r_{-}$, Fragilaria virescens $=$ $F r_{-} v$, Goniochloris $s p=$ Gon, Gomphonema $s p=$ Gom, Gyrosigma spencerii $=G y_{-} s$, Mastogloia baltica $=$ Ma_b, Navicula sp2 $=$ Nav2, Nitzschia intermedia $=N i \_i$, Nitzschia ignorata $=N i \_n$, Nitzschia periata $=N i \_p$, Palmella $\mathrm{sp}=$ Pam, Phacus $\mathrm{sp}=$ Pha, Phaeocystis $\mathrm{sp}=$ Pha, Pleurotaenium cylindrium $=P r_{-} c$, Pleurotaenium excelsum $=$ Pr_e, Stenopterobia ancepts $=S t \_$, Surirella spiralis $=$Sur, Trachelomonas conica $f$ punctata $=$ $T r_{-} c$, Trachelomonas olonga $=T r_{-} o$, Amphora coffeaeformis $=$ Am_c, Anacystic sp2 $=$ Ana1, Cosmarium clepsydra var dissimile $=C m_{-} c$, Cyclotella glomerata $=C y_{-} g$, Navicula $\mathrm{sp}=$ Nav, Trachelomonas volzii $=T r_{-}$, Chlorobacterium aggregatum $=C I_{-}$, Diatoma elongatum $=$ Di_e, Fragilaria heidenii $=F r_{-} h$, Fragilaria heidenii $=F r_{-} h$, Cryptomonas sp = Cry, Goniochloris sp2 = Gon2, Goniochloris sp3 = Gon3, Microceleus $\mathrm{sp}=$ Mic, Leptocylindricus danicus $=$ Le_d, Licmophora $\mathrm{sp}=$ Lic, Nitzschia flexoides $=$ Ni_f, Nitzschia frustulum var bunheimiana $=$ Ni_f, Scenedesmus $\mathrm{sp}=S c e$, Stenopterobia $\mathrm{sp}=$ Ste, Stichococcus $\mathrm{sp}=$ St $i$, Synedra acus var radians $=S n_{-}$. .

benthic algal diversity and Richness $(r=0.82$ and $\mathrm{P}=0.024)$. There was a negative correlation between benthic diversity and Organic Carbon $(\mathrm{r}=-0.844$ and $\mathrm{P}$ $<0.05)$ benthic diversity and Organic Matter $(\mathrm{r}=-0.846, \mathrm{P}<0.05)$, benthic richness and available phosphorus $(\mathrm{r}=-0.785, \mathrm{P}<0.05)$, Base saturation and $\mathrm{K}$ $(\mathrm{r}=-0.916, \mathrm{P}<0.05)$.

Table 10 shows the correspondence analyses between nutrient and benthic algal diversity parameters across sites. The first two components explain $93.84 \%$ of the total inertia (Qual $=0.976$ and 0.951 respectively). Analysis of the contingency 
Table 10. Simple Correspondence Analysis of benthic occurrence and nutrients.

\begin{tabular}{cccccccc}
\hline \multicolumn{7}{c}{ Analysis of contingency table } \\
\hline Axis & 1 & 2 & 3 & 4 & 5 & 6 & Total \\
\hline Inertia & 0.0156 & 0.0011 & 0.0007 & 0.0002 & 0.0001 & 0 & 0.0178 \\
Proportion & 0.8773 & 0.0611 & 0.0409 & 0.0134 & 0.0065 & 0.0008 & \\
Cumulative & 0.8773 & 0.9384 & 0.9793 & 0.9927 & 0.9992 & 1 & \\
\hline & & Row Contribution & & & \\
\hline Name & Qual & \multicolumn{7}{c}{ Corr } & Contr & Corr & Contr & \\
Site 1 & 0.951 & 0.946 & 0.245 & 0.005 & 0.019 & \\
Site 2 & 0.976 & 0.887 & 0.183 & 0.089 & 0.263 & \\
Site 3 & 0.934 & 0.917 & 0.093 & 0.016 & 0.024 & \\
Site 4 & 0.882 & 0.19 & 0.007 & 0.692 & 0.357 & \\
Site 5 & 0.904 & 0.826 & 0.157 & 0.078 & 0.212 & \\
Site 6 & 0.799 & 0.708 & 0.066 & 0.091 & 0.122 & \\
Site 7 & 0.982 & 0.981 & 0.25 & 0.001 & 0.002 & \\
\hline
\end{tabular}

Qual = the proportion of the row inertia represented by the two components. Coord $=$ the principal coordinates of the rows. Corr $=$ the contribution of the component to the inertia of the row. Contr $=$ the contribution of each row to the axis inertia, Inert $=$ the proportion of the total inertia contributed by each row.

table indicated that Components 1 and 2 contributed $87.73 \%$ and $6.11 \%$ respectively of the total inertia. Component 1 best explains the patterns in Site 1, Site 3, Site 5 and Site 7 in terms of physicochemical and benthic parameters, while the patterns in the rest of the sites are best explained by Component 2 .

\section{Discussion}

Mangroves play a critical role in the ecology and economy of coastal communities. In Tiko like elsewhere in Cameroon, electricity and gas supplies are epileptic and expensive. The population therefore depends on biomass for energy. In the current study, Rhizophora mangle (red mangrove) was the most used species, not just because it dominates the mangroves of Tiko, but more because of its wood qualities. Rhizophora mangle produces high density red wood suitable for cooking, smoking, drying, roasting and charcoal production. Din [40] and Feka [18] reported heavy exploitation of the same species in neighboring towns of Douala and South West Region respectively for the same uses. In this fishing area void of steady electricity supply, fish smoking has become a huge business linking the fishermen and the consumers, at the cost of the mangrove ecosystem. Settlements within the mangrove mean permanent destruction of the vegetation, waste disposal, agriculture, wood extraction (for fuel and construction) among others. All these perturbations impact the physicochemical and biological components of the ecosystem.

Sediment texture in all study sites within the Tiko Mangrove was predomi- 
nantly clayey, similar to the observations of [41] [42] [43]. High clay content in estuarine mangrove can be explained by their alluvial origins which in a sedimentary environment would result in accumulation of fine particles [17]. Contrary to this study, [44] reported a sediment texture of silty loam in a naturally generated mangrove of Southern Gulf of Kachchh, Gujarat, India while [45] observed a highly dominant fraction of sand in the Red Sea coast of Saudi Arabia. Sediments in the study area receive effluent from plantations, industrial discharges, urban and domestic discharges. The low $\mathrm{pH}$ in all sites may be as a result of the application of acid-forming fertilizers such as urea which might be leaching into the mangrove swamps [46]. The very low $\mathrm{pH}$ (2.9) at Site 7 could also be as a result of synergy of biological activity in disturbed mangrove under inundation [47] and nearness of this site to the rubber factory (about $200 \mathrm{~m}$ ) which releases effluent that may be rich in acidic substances (sulfuric acid) used in processing rubber.

The organic carbon content represents the organic matter in the sediments which is of potential significance for mangrove productivity. Five percent (5\%) of organic carbon is ideal for soil composition in mangrove [48]. Martin [49] also reported high levels of organic carbon (above 5\%) in the estuary of India and attributed this high level to environmental deterioration. Spatial variations in organic carbon have been attributed to sediment temperature fluctuations, nature of vegetation, soil texture, and rate of accumulation of dead and decayed foliage, topography, and water depth [48]. Sediment chemistry varied across sites. The high concentration of $\mathrm{Mg}$ could be due to combination of amphiboles, olivine, dolomite, clay and anthropogenic activities [50]. The values of the major cations ( $\mathrm{Ca}, \mathrm{Mg}, \mathrm{Na}$ and $\mathrm{K}$ ) showed a lot of variations in the soils in this study across sites. Ca levels ranged between 2.48 and $8.08 \mathrm{meq} / 100 \mathrm{~g}$. According to [51], Ca levels in wetland soils were influenced by benthic algal species especially the Bacillariophyta which use Ca for cell wall formation. High ECEC implies that nutrients are retained in the sediments and there is little leaching and transportation to the water systems. Soils which are low in ECEC are said to have low nutrient retention capacities and nutrients will therefore be easily transported in to the water systems [52].

Changes in vegetation cover have been shown to influence algal community [9]. The abundance of diatoms is an indication of high nutrient status of the sites. All seven sites recorded Mcrocystis sp. This indicates that the sediments are polluted because Microcystis sp is an indicator of environmental pollution [26]. The nutrient levels of the study sites were high which might be the reason for Microcystis being the most dominant species. This is in line with the results of Homma [52] who reported that the dominance of toxic Microcystis is promoted by high nutrient concentrations. Scenedesmus sp was recorded during this study, indicating eutrophic conditions [53]. The presence of Chroodactylon $\mathrm{sp}$ (red algae), in this system is of concern as they are known to produce harmful algal blooms.

Results obtained from simple correspondence analysis showed that some 
benthic species were associated to particular sites. The association of these species to particular sites could be related to the activities of these sites, the soil physical and chemical properties, and nutrients levels. Similar findings were reported by [18] [54], who found that physiochemical parameters had a direct relationship with some benthic species such as Navicula viridula, Navicula symmetrica, Nitzschia palae. They also reported that Nitzschia palae is associated with high nutrient soils. Stancheva [55] reported that Cladophora glomerata indicates high total $\mathrm{N}$ levels and Leptolyngbya foveolara is associated with high available P levels.

The lack of relationship with P load and benthic diversity in the current study suggests that $\mathrm{P}$ is not a limiting factor for benthic growth in this area. Lavoie [56] found that $\mathrm{P}$ enrichment does not appear to have any positive effect on benthic algal growth. A negative relationship was observed between species diversity with organic Carbon and organic matter, species richness and available $\mathrm{P}$. This negative relationship implies that as organic carbon and matter levels increase species diversity reduces.

\section{Conclusion}

The high productivity and proximity of the mangroves of Tiko to the urban center has favoured settlement in and near these forests. Anthropogenic activities such as wood extraction and transportation, fish smoking, charcoal production, coastal industrialization, and conversion to agricultural land and settlement adversely affect dispersion, establishment and growth dynamics of these forests. These perturbations impact the physicochemical parameters of the sediments, with consequences on the algal community, the basis of the food chain. The diversity and abundance of the benthic algae showed a polluted environment, with Microsystis, Prorocentrum, Oscillatoria and others indexed.

\section{Recommendations}

Monitoring and assessment of benthic blooms is of vital importance in conservation and management of the Tiko mangroves.

\section{Acknowledgements}

The authors are grateful to the Life Sciences Laboratory of the University of Buea for algal analyses and the Soil and Environmental Chemistry Laboratory of the University of Dschang for the physicochemical analyses.

\section{Conflicts of Interest}

I here declare that there is no conflict of interest among the authors.

\section{References}

[1] Daza, D.A.V., Moreno, H.S., Portz, L., Manzolli, R.P., Bolívar-Anillo, H.J. and Anfuso, G. (2020) Mangrove Forests Evolution and Threats in the Caribbean Sea of 
Colombia. Water, 12, 1113. https://doi.org/10.3390/w12041113

[2] Fotabong-Atheull, A.N., Din, N., Longonje, S.N., Koedam, N. and Dahdouh-Guebas, F. (2009) Commercial Activities and Subsistence Utilization of Mangrove Forests around the Wouri Estuary and the Douala-Edea Reserve (Cameroon). Journal of Ethnobiology and Ethnomedicine, 5, 35. https://doi.org/10.1186/1746-4269-5-35

[3] Jiazet, D.H.J. (2019) Potential Impact of Fish Smoking on Mangrove Resources in Southwest Cameroon. Tropical Conservation Science, 12, 1-13. https://doi.org/10.1177/1940082919833300

[4] Obade, P.T., Koedam, N., Soetaert, K., Neukermans, G., Bogaert, J., et al. (2009) Impact of Anthropogenic Disturbance on a Mangrove Forest Assessed by a 1D Cellular Automaton Model Using LOTKA-VOLTERRA-TYPE Competition. International Journal of Design \& Nature and Ecodynamics, 3, 296-320.

[5] Shibabaw, T., Beyene, A., Awoke, A., Tirfie, M., Azage, M. and Triest, L. (2021) Diatom Community Structure in Relation to Environmental Factors in Human Influenced Rivers and Streams in Tropical Africa. PLOS ONE, 16, e0246043.

https://doi.org/10.1371/journal.pone.0246043

[6] Hendrarto, I.B. and Nitisupario, M. (2011) Biodiversity of Benthic Diatom and Primary Productivity of Benthic Micro-Flora in Mangrove Forests on Central Java. Journal of Coastal Development, 14, 131-140.

[7] Muro-Torres, V.M., Amezcua, F., Soto-Jiménez, M., Balart, E.F., Serviere-Zaragoza, E., Green, L. and Rajnohova, J. (2020) Primary Sources and Food Web Structure of a Tropical Wetland with High Density of Mangrove Forest. Water, 12, 3105. https://doi.org/10.3390/w12113105

[8] Kon, K., Kurokura, H. and Hayashizak, K. (2007) Role of Microhabitats in Food Webs of Benthic Communities in a Mangrove Forest. Marine Ecology Progress Series, 340, 55-62. https://doi.org/10.3354/meps340055

[9] Xia, L., Zhu, Y. and Zhao, Z. (2020) Understanding the Ecological Response of Planktonic and Benthic Epipelic Algae to Environmental Factors in an Urban Rivers System. Water, 12, 1311. https://doi.org/10.3390/w12051311

[10] Fonge, B.A., Tening, A.S., Egbe, E.A., Yinda, G.S., Fongod, A.N. and Achu, R.M. (2012) Phytoplankton Diversity and Abundance in Ndop Wetland Plain, Cameroon. African Journal of Environmental Science and Technology, 6, 247-257. https://doi.org/10.5897/AJEST12.025

[11] Carugati, L., Gatto, B., Rastelli, E., Martire, M.L., Coral, C., Silvestro Greco, S. and Danovaro, R. (2018) Impact of Mangrove Forests Degradation on Biodiversity and Ecosystem Functioning. Scientific Reports, 8, Article No. 13298.

https://doi.org/10.1038/s41598-018-31683-0

[12] Newell, R.I.E., Marshall, N., Sasekumar, A. and Chong, V.C. (1995) Relative Importance of Benthic Microalgae, Phytoplankton, and Mangroves as Sources of Nutrition for Penaeid Prawns and Other Coastal Invertebrates from Malaysia. Marine Biology, 123, 595-606. https://doi.org/10.1007/BF00349238

[13] Gao, Y. and Lin, G. (2018) Algal Diversity and Their Importance in Ecological Processes in Typical Mangrove Ecosystems. Biodiversity Science, 26, 1223-1235. https://doi.org/10.17520/biods.2018080

[14] Nfotabong-Atheull, A., Din, N., Koum, L.G.E., Satyanarayana, B., Koedam, N. and Dahdouh-Guebas, F. (2011) Assessing Forest Products Usage and Local Residents' Perception of Environmental Changes in Peri-Urban and Rural Mangroves of Cameroon, Central Africa. Journal of Ethnobiology and Ethnomedicine, 7, Article No. 41. http://www.ethnobiomed.com/content/7/1/41 
https://doi.org/10.1186/1746-4269-7-41

[15] Ajonina, G.N. (2008) Inventory and Modeling Mangrove Forest Stand Dynamics Following Different Levels of Exploitation Pressure in the Douala-Edea Atlantic Coast of Cameroon, Central Africa. Ph.D. Thesis, Albert-Ludwigs-Universitat, Freburg IM Breisgau, $215 \mathrm{p}$.

[16] Simon, L.N. and Raffaelli, D. (2012) Assessing Ecosystem Effects of Small Scale Cutting of Cameroon Mangrove Forests. Journal of Ecology and the Natural Environment, 4, 126-134. https://doi.org/10.5897/JENE11.131

[17] Ngole-Jeme, V.M., Fonge, B.A., Tabot, P.T. and Mumbang, C. (2016) Impact of Logging Activities in a Tropical Mangrove on Ecosystem Diversity and Sediment Heavy Metal Concentrations. Journal of Coastal Conservation Planning and Management, 20, 245-255. https://doi.org/10.1007/s11852-016-0435-y

[18] Feka, N.Z. and Manzano, M.G. (2008) The Implications of Wood Exploitation for Fish Smoking on Mangrove Ecosystem Conservation in the South West Province, Cameroon. Tropical Conservation Science, 1, 222-241. https://doi.org/10.1177/194008290800100305

[19] Vincent, F.A. (2005) Fako Local Economic Development Agency. Sub-Title: Project to Alleviate Poverty through Self-Employment and Enterprise Creation in Fako Division of Cameroon.

[20] Cameroon Mangrove Conservation Network (CMN) (2007) Chapter of the African Mangrove Network (AMN). Matanda News, to Inform and Educate the Cameroonian Public on Mangrove Conservation Issues. Vol. 1 No. 1.

[21] Egbe, A.E., Tabot, P.T., Fonge, B.A. and Bechem, E. (2012) Simulation of the Impacts of Three Management Regimes on Carbon Sinks in Rubber and Oil Palm Plantation Ecosystems of South-Western Cameroon. Journal of Ecology and the Natural Environment, 4, 154-162. https://doi.org/10.5897/JENE11.146

[22] Reddy, K.R., Wang, Y., DeBusk, W.F., Fisher, M.M. and Newman, S. (1998) Forms of Soil Phosphorus in Selected Hydrologic Units of the Florida Everglades. Soil Science Society of American Journal, 62, 1134-1147. https://doi.org/10.2136/sssaj1998.03615995006200040039x

[23] Walkley, A. and Black, I.A. (1934) An Examination of Degtjareff Method for Determining Soil Organic Matter and a Proposed Modification of the Chromic Acid Titration Method. Soil Science, 37, 29-37. https://doi.org/10.1097/00010694-193401000-00003

[24] IITA (1979) Selected Methods for Soil and Plant Analysis. International Institute of Tropical Agriculture, Manual Series, No. 1.70 p.

[25] Danielson, T.J. (2014) Protocols for Sampling Algae in Wadeable Rivers, Streams, and Freshwater Wetlands. Maine Department of Environmental Protection DEPLW-0634B-2014, 19 p.

[26] Bellinger, E.G. and Sigee, D.C. (2010) Freshwater Algae. Identification and Use as Bioindicators. Wiley-Blackwell, Hoboken, $271 \mathrm{p}$. https://doi.org/10.1002/9780470689554.ch1

[27] Compere, P. (1977) Algues de la region du Tchad. Cah. ORSTOM, Ser. Hydrobiology, 11, 77-177.

[28] Lltis, A. (1980) Les Algues, in Flore et Faune aquatiques de l'Afrique Sahelo-Soudanienne. Orstom, 1, 9-61.

[29] Gasse, F. (1986) East Africa Diatoms: Taxonomy, Ecological Distribution. Bibliotheca Diatomologica, 11, 201. https://doi.org/10.1007/978-94-009-4808-2 12 
[30] Krammer, K. and Lange-Bertalot, H. (1986) Susswasserflora of Central Europe. Bacillariophyceae. Part 1: Naviculaceae. Oxford University Press, Heiderlberg, 876.

[31] Krammer, K. and Lange-Bertalot, H. (1988) Susswasserflora of Central Europe. Bacillariophyceae. Part 3: Centrales Fragilariaceae, Eunotiaceae. VEB Gustave F. Fischer Verlag, Jena, 576.

[32] Krammer, K. and Lange-Bertalot, H. (1991) Susswasserflora of Central Europe. Bacillariophyceae. Part 4: Achnanthaceae, Critical Additions to Navicula (Lineolatae) and Gomphonema. Gustav Fisher Verlag, Jena, 447.

[33] Krammer, K. and Lange-Bertalot, H. (2002) Baccillariophyceae. Part 3: Centraceae, Fragrariaceae, and Eunotiaceae. Gustav Fisher Verlag, Jena, 3365.

[34] Catherine, G.M., Orcutt, D.M., Kreeger, D.A., Parker, B.C., Jones, V.A. and Neves, R.J. (2003) Biochemical Composition of Three Algal Species Proposed as Food for Captive Freshwater Mussels. Journal of Applied Phycology, 15, 1-11. https://doi.org/10.1023/A:1022929423011

[35] Nwankwo, E. and Onyema, I.C. (2003) A Checklist of Planktonic Algae off Lagos Coast. Journal of Scientific Research and Development, 9, 75-85.

[36] Nwankwo, D.I. (2003) A Practical Guide to the Study of Algae. JAS Publishers, Lagos, 84 .

[37] Nguetsop, V.F., Fonkou, U.T., Assah, V.M.D., Nangtson, M.N. and Pinta, J.Y. (2007) Relationships between Algae Taxa and Physicochemical Characteristics of Water in Wetlands and Water Bodies. Cameroon Journal of Experimental Biology, 3, 70-79. https://doi.org/10.4314/cajeb.v3i2.37965

[38] Guiry, M.D. (2013) Algae Base. World-Wide Electronic Publication, National University of Ireland, Galway. http://www.algaebase.org

[39] Guiry, M.D. and Guiry, G.M. (2019) AlgaeBase. World-Wide Electronic Publication, National University of Ireland, Galway. http://www.algaebase.org

[40] Din, N., Saenger, P., Jules, P.R., Siegfried, D.D. and Basco, F. (2008) Logging Activities in Mangrove Forests: A Case Study of Douala Cameroon. African Journal of Environmental Science and Technology, 2, 22-30.

[41] Ferreira, T.O., Otero, X.L., de Souza Junior, V.S., Vidal-Torrado, P., Macías, F. and Firme, L.P. (2010) Spatial Patterns of Soil Attributes and Components in a Mangrove System in Southeast Brazil (São Paulo). Journal of Soils and Sediments, 10, 995-1006. https://doi.org/10.1007/s11368-010-0224-4

[42] Almulla, L. (2013) Soil Site Suitability Evaluation for Mangrove Plantation in Kuwait. World Applied Sciences Journal, 22, 1644-1651.

[43] Manikandan, R. and Vijayakumar, R. (2016) Physico-Chemical Analysis of Marine Soils From Different Ecosystem Palk Strait Coastal Regions of Tamilnadu, India. Journal of Marine Science: Research and Development, 6, 208. https://doi.org/10.4172/2155-9910.1000208

[44] Das, L., Patel, R., Salvi, H. and Kamboj, R.D. (2019) Assessment of Natural Regeneration of Mangrove with Reference to Edaphic Factors and Water in Southern Gulf of Kachchh, Gujarat, India. Heliyon, 5, e02250. https://doi.org/10.1016/j.heliyon.2019.e02250

[45] Alzahrani, D.A., Selim, El.-M.M. and El-Sherbiny, M.M. (2018) Ecological Assessment of Heavy Metals in the Grey Mangrove (Avicennia marina) and Associated Sediments along the Red Sea Coast of Saudi Arabia. Oceanologia, 60, 513-526. https://doi.org/10.1016/j.oceano.2018.04.002

[46] Davari, A., Danehkar, A., Nematolah, K. and Hadi, P. (2010) Heavy Metal Conta- 
mination of Sediments in Mangrove Forests of the Persian Gulf. Journal of Food, Agriculture and Environment, 8, 1280-1284.

[47] Oxmann, J.F., Pham, Q.H., Schwendenmann, L., Stellman, J.M. and Lara, R.J. (2010) Mangrove Reforestation in Vietnam: The Effect of Sediment Physicochemical Properties on Nutrient Cycling. Plant Soil, 326, 225-241. https://doi.org/10.1007/s11104-009-0003-4

[48] Muhibbullah, M., Nurul, A.S. and Chowdhury, M.A.T. (2005) Some Physicochemical Parameter of Soil and Water of Sundarban Mangrove Forest, Bangladesh. Journal of Biological Science, 5, 354-357. https://doi.org/10.3923/jbs.2005.354.357

[49] Martin, G.D., Nisha, P.A., Balachandran, K.K., Madhu, N.V., Nair, M., Shaiju, P., Joseph, T., Srinivas, K. and Gupta, G.V.M. (2011) Eutrophication Induced Changes in Benthic Community Structure of a Flow-Restricted Tropical Estuary (Cochin Backwaters), India. Environmental Monitoring and Assessment, 176, 427-438. https://doi.org/10.1007/s10661-010-1594-1

[50] Sahoo, K., Jee, P.K., Dhal, N.K. and Das, R. (2017) Physico-Chemical Sediment Properties of Mangroves of Odisha, India. Journal of Oceanography and Marine Resources, 5, 162. https://doi.org/10.4172/2572-3103.1000162

[51] Tening, A.S., Chuyong, G.B., Asongwe, G.A., Fonge, B.A., Lifongo, L.L., Mvondo-Ze, A.D., Che, V.B. and Suh, C.E. (2013) Contribution of Some Water Bodies and the Role of Soils in the Physicochemical Encrichment of the Douala-Edea Mangrove Ecosystem. African Journal of Environmental Science and Technology, 7, 336-349.

[52] Fonge, B.A., Tening, A.S., Achu, R.M. and Yinda, G.S. (2013) Effects of Physico-Chemical Parameters on the Diversity and Abundance of Benthic Algae in an Agricultural Wetland in Ndop Plain, Cameroon. Global Advanced Research Journal of Agricultural Science, 2, 217-230. http://garj.org/garjas/index.htm

[53] Homma, T., Komatsu, N., Negishi, M., Katagami, Y., Nakamura, K. and Park, H.D. (2008) Influence of Dissolved Inorganic Nitrogen and Phosphorus Concentration on the Horizonta and Temporal Changes of Microcystis Population in Lake Kitaua. Proceeding of Taal 2007: The 12th World Lake Conference, Jaipur, 28 October-2 November 2006, 1423-1429.

[54] Yana, E. and Peerapornpisal, Y. (2009) Diversity of Benthic Algae and Water Quality in Tributaries of the Mekong River Passing Thailand and Some Parts of Lao PDR. KKu Science Journal, 37, 30-41.

[55] Stancheva, A.R., Fetscher, E., and Sheath, R.G. (2012) A Novel Quantification Method for Stream-Inhabiting, Non-Diatom Benthic Algae, and Its Application in Bioassessment. Hydrobiologia, 684, 225-239.

https://doi.org/10.1007/s10750-011-0986-8

[56] Lavoie, I., Warwick, F.V., Reinhard, P. and Painchaud, J. (2004) Benthic Algae as Bioindicators of Agricultural Pollution in the Streams and Rivers of Southern Quebec (Canada). Aquatic Ecosystem Health and Management, 7, 43-58.

https://doi.org/10.1080/14634980490281236 